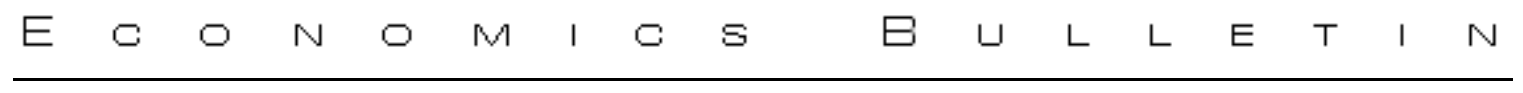

\title{
A Comparative Analysis of IPO Proceeds Under Alternative Regulatory Environments: A Comment.
}

\author{
Neil Brisley \\ Finance Area Group, Richard Ivey School of Business, University of Western Ontario
}

\begin{abstract}
We clarify and reinterpret the results of Benveniste and Wilhelm (1990) concerning the effect of a uniform price restriction on the proceeds of an IPO. If regular institutional investors are, on average, at least as well informed as ordinary retail investors then our corrected version of Benveniste and Wilhelm's model shows that a uniform price restriction does not affect IPO proceeds.
\end{abstract}

\footnotetext{
I am grateful for the support of an "MBA Class of "89 Faculty Fellowship". Whilst not part of my dissertation, this note arose from a PhD seminar taken in March 1998 at INSEAD, Fontainebleau, France. For this reason I also gratefully acknowledge doctoral scholarships from INSEAD and the Sasakawa Foundation.

Citation: Brisley, Neil, (2003) "A Comparative Analysis of IPO Proceeds Under Alternative Regulatory Environments: A

Comment.." Economics Bulletin, Vol. 7, No. 1 pp. 1-7

Submitted: October 11, 2002. Accepted: January 13, 2003.

URL: http://www.economicsbulletin.com/2003/volume7/EB-02G20005A.pdf
} 


\section{Introduction}

Benveniste and Wilhelm (1990) (hereafter B\&W) address the problem of an Initial Public Offer where the issuer uses the bookbuilding method to elicit information from potentially informed investors. Their contribution is of importance in the debate over whether regulatory constraints on the ability of underwriters to price discriminate may affect the proceeds attainable from the issue. $\mathrm{B} \& \mathrm{~W}$ contend that a uniform price restriction may increase the cost of soliciting information from regular investors, thus reducing the proceeds available to the issuer. However, the model of B\&W would appear to contain several inconsistencies. In this Comment, we attempt to clarify their results and reinterpret their conclusions. Our corrections impact dramatically upon the statements of their central Theorem 1 and Proposition 1. Specifically, using B\&W's assumptions, we obtain a very different result for the magnitude of underpricing offered to retail investors. In turn, this alters the circumstances under which the issuer will find it optimal to offer shares to retail investors. Only when regular institutional investors are, on average, less well informed than retail investors may the uniform pricing constraint bind. Otherwise, a uniform price restriction does not affect IPO proceeds.

\section{Model set-up}

B\&W's analysis " $\ldots$ is carried out in the context of a model in which both regular and retail investors may possess information that the underwriter does not. Within this environment, the underwriter conditions the offer price and allocation on information gathered in the premarket and fully allocates the issue to regular or retail investors before the aftermarket price conveys the added information".

The State of the Environment can be $g, b$ (good/bad information exists) or $u$ (no information exists), with probabilities $\frac{\pi}{2}, \frac{\pi}{2}$ and $1-\pi$ respectively. In the first stage of the marketing effort, "... the underwriter attempts to learn investors' information by soliciting indications of interest from regular investors." We refer the reader to the original article for fuller details of the model.

Regular Investors Conditioned on information existing, the probability that any particular regular investor has this information is $\pi_{i}$ and each regular investor is independent of the others.

$\mathrm{B} \& \mathrm{~W}$ state that "...conditioned on being uninformed, a regular investor faces the probability $P(u, 0)$ that all other regulars are uninformed, where

$$
P(u, 0)=\frac{\pi}{2}[P(g, 0)+P(b, 0)]+(1-\pi)
$$

(B\&W, equation 4, page 180)

This expression would appear to be incorrect. 
The probabilities $\frac{\pi}{2}, \frac{\pi}{2}$ and $1-\pi$ are ex-ante unconditional probabilities for the possible states of the world, $g, b$ and $u$. However, when a regular finds himself to be uninformed, he should update these probabilities using Bayes' Rule to take account of his (absence of) information.

The conditional probabilities are:

$$
\begin{gathered}
\operatorname{Pr}(\text { state } g \mid \text { uninformed })=\frac{\frac{\pi}{2}\left(1-\pi_{i}\right)}{(1-\pi)+\pi\left(1-\pi_{i}\right)}=\frac{\frac{\pi}{2}\left(1-\pi_{i}\right)}{1-\pi \pi_{i}} \\
\operatorname{Pr}(\text { state } b \mid \text { uninformed })=\frac{\frac{\pi}{2}\left(1-\pi_{i}\right)}{1-\pi \pi_{i}} \\
\operatorname{Pr}(\text { state } u \mid \text { uninformed })=\frac{1-\pi}{1-\pi \pi_{i}}
\end{gathered}
$$

The correct expression for this conditional probability is therefore:

$$
P(u, 0)=\frac{\frac{\pi}{2}\left(1-\pi_{i}\right)[P(g, 0)+P(b, 0)]+(1-\pi)}{1-\pi \pi_{i}}
$$

B\&W correctly note that (page 180) "...the unconditional and conditional probabilities are related by

$$
P(g, h)=\frac{H}{(H-h)} \frac{\pi}{2}\left(1-\pi_{i}\right) P(g, h)
$$

from which

$$
\frac{\pi}{2}\left(1-\pi_{i}\right) P(g, 0)=P(g, 0)=\frac{\pi}{2}\left(1-\pi_{i}\right)^{H}=P(b, 0)=\frac{\pi}{2}\left(1-\pi_{i}\right) P(b, 0)
$$

thus we can rewrite equation (5) explicitly as

$$
P(u, 0)=\frac{\pi\left(1-\pi_{i}\right)^{H}+(1-\pi)}{1-\pi \pi_{i}}
$$

Retail Investors $\mathrm{B} \& \mathrm{~W}$ Theorem 1 derives the expected underpricing required by uninformed retail investors when all $H$ regulars have indicated 'uninformed'. In their proof (Appendix 1), B\&W appear to weight the state dependant payoffs with the unconditional probabilities of each state. However, since the expected underpricing in Theorem 1 arises only in situations when all $H$ regulars have said 'uninformed', it should be the conditional probabilities (of true states $g, b$ and $u$, given that $H$ regulars have said 'uninformed') that are used to reflect this information.

Theorem 1 would therefore appear to be incorrect.

Noting

$$
P(g, 0)=P(b, 0)=\frac{\pi}{2}\left(1-\pi_{i}\right) P(g, 0)=\frac{\pi}{2}\left(1-\pi_{i}\right)^{H}
$$

and

$$
P(u, 0)=1-\pi
$$


then the conditional probabilities are

$$
\begin{aligned}
& \operatorname{Pr}(\text { state } g \mid H \text { are uninformed })=\frac{\frac{\pi}{2}\left(1-\pi_{i}\right)^{H}}{\pi\left(1-\pi_{i}\right)^{H}+1-\pi} \\
& \operatorname{Pr}(\text { state } b \mid H \text { are uninformed })=\frac{\frac{\pi}{2}\left(1-\pi_{i}\right)^{H}}{\pi\left(1-\pi_{i}\right)^{H}+1-\pi}
\end{aligned}
$$

and

$$
\operatorname{Pr}(\text { state } u \mid H \text { are uninformed })=\frac{1-\pi}{\pi\left(1-\pi_{i}\right)^{H}+1-\pi}
$$

and so expression (A1) on page 200 should read

$$
\left(\begin{array}{c}
\frac{\pi}{2}\left(1-\pi_{i}\right)^{H}\left[s^{g}-\left(s^{u}-\varepsilon\right)\right] \beta_{u} Q_{r} \\
+(1-\pi)\left[s^{u}-\left(s^{u}-\varepsilon\right)\right] \beta_{u} Q_{r} \\
+\frac{\pi}{2}\left(1-\pi_{i}\right)^{H}\left[s^{b}-\left(s^{u}-\varepsilon\right)\right] Q_{r}
\end{array}\right)=0
$$

Substituting

$$
s^{g}-s^{u}=s^{u}-s^{b}=\alpha
$$

we obtain the solution for the magnitude of underpricing (for derivation see appendix)

$$
\varepsilon=\frac{\alpha\left(1-\beta_{u}\right)}{1+\beta_{u}+\frac{2 \beta_{u}(1-\pi)}{\pi\left(1-\pi_{i}\right)^{H}}}
$$

This expression should replace BE⿱SW's equation (5), page 181. B\&W's equation (6) is affected accordingly and we offer a corrected version in the appendix.

We see that as $H$ increases, so $\varepsilon$ decreases corresponding to the intuition that the larger the pool of regular investors, the more reliable is a unanimous 'No Information' declaration and so the smaller is the Rock Discount required to protect uninformed retail investors. We note that B\&W's $\varepsilon$ is independent of $H$ and $\pi_{i}$ and so does not possess this intuitive property.

A similar error of principle seems to be contained in equations (9) and (10) (page 183) and we offer corrected versions in the appendix. BE⿱ $W$ appear to correctly update when information is $g$, yet they fail to update correctly when information is $u$.

The error in the expression for $\varepsilon$ in Theorem 1 is carried through to the derivation of Proposition 1 which states the condition for implementing Strategy 1 (allocating the entire issue to regular investors when all $H$ have said 'uninformed'). Substituting our corrected $\varepsilon$ into the inequality at the bottom of page 202, the condition for Strategy 1 to be optimal becomes

$$
\frac{H P(., 0)}{P(g, 1)} \geqslant \frac{\pi\left(1-\pi_{i}\right)^{H}\left(\beta_{u}+1\right)+2 \beta_{u}(1-\pi)}{\left(1-\beta_{u}\right) \pi\left(1-\pi_{i}\right)^{H}}
$$

This expression should replace BEGW's equation (15), page $18 \%$. 
By substituting in the explicit expressions

$$
P(., 0)=\pi\left(1-\pi_{i}\right)^{H}+(1-\pi)
$$

and

$$
P(g, 1)=\frac{\pi}{2} H \pi_{i}\left(1-\pi_{i}\right)^{H-1}
$$

we can simplify this to (for derivation see appendix)

$$
\pi\left(1-\pi_{i}\right)^{H}\left(3-\beta_{u}\right)\left[\frac{2\left(1-\beta_{u}\right)}{\left(3-\beta_{u}\right)}-\pi_{i}\right]+2(1-\pi)\left[\left(1-\beta_{u}\right)-\pi_{i}\right] \geqslant 0
$$

which should replace BESW's equation (17).

Thus we can now derive explicit bounds on the relationships between the parameters $\pi, \pi_{i}, \beta_{u}$, and $H$, which determine whether Strategy 1 is optimal or not. The alternative, Strategy 2, involves allocating the entire issue to retail investors when all $H$ regulars have said 'uninformed'.

Specifically, if

$$
\pi_{i} \leq \frac{2\left(1-\beta_{u}\right)}{\left(3-\beta_{u}\right)}
$$

then

$$
\left[\left(1-\beta_{u}\right)-\pi_{i}\right]>0
$$

Hence our equation (21) provides a sufficient, but not necessary, condition for Strategy 1 to be optimal.

Moreover, if

$$
\pi_{i} \geq 1-\beta_{u}
$$

then

$$
\frac{2\left(1-\beta_{u}\right)}{\left(3-\beta_{u}\right)}-\pi_{i}<0
$$

Hence our equation (23) provides a sufficient, but not necessary, condition for Strategy 2 to be optimal.

Condition (23) has a very intuitive interpretation: if information exists, then $1-\beta_{u}$ is the proportion of retail investors who will be informed and $\pi_{i}$ is the probability that any particular regular investor will be informed. Thus a sufficient, but not necessary, condition for Strategy 2 to be optimal is that Regular Investors are, on average, at least as well-informed as retail investors.

It is only in the interval,

$$
\pi_{i} \in\left(\frac{2-2 \beta_{u}}{3-\beta_{u}}, 1-\beta_{u}\right)
$$

that the values of $\pi$ and $H$ are decisive in choosing between strategies.

Figure 1 illustrates the possibilities: Above the straight line, Strategy 2 is optimal. Below the curve, Strategy 1 is optimal. In between the line and the curve, Strategy 1 is optimal if and only if

$$
\pi\left(1-\pi_{i}\right)^{H} \leq \frac{2(1-\pi)\left[1-\pi_{i}-\beta_{u}\right]}{\left[4-\left(3-\beta_{u}\right)\left(2-\pi_{i}\right)\right]}
$$


Figure 1: Graphs of $\pi_{i}=\frac{2\left(1-\beta_{u}\right)}{\left(3-\beta_{u}\right)}$ and $\pi_{i}=1-\beta_{u}$

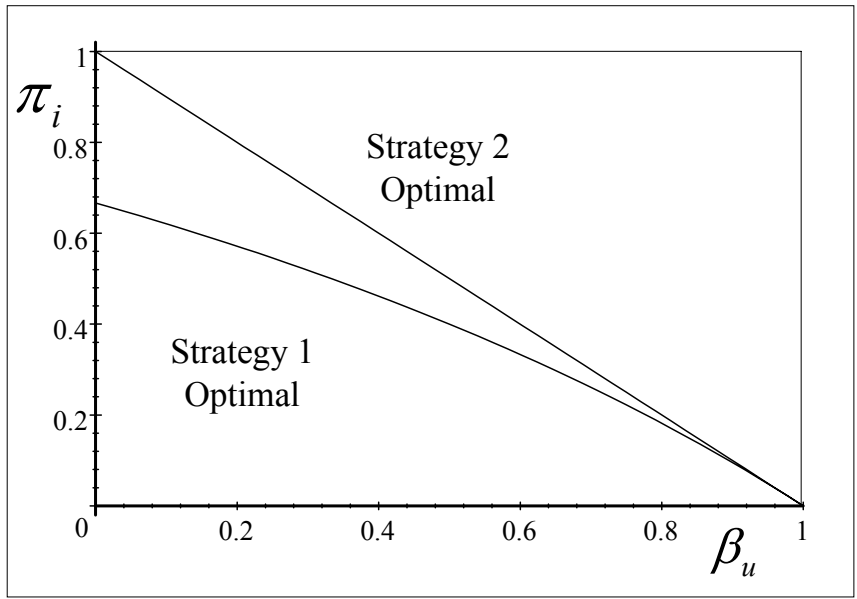

i.e. when $\pi$ is 'small enough' (small probability of information existing) or when $H$ is 'high enough' (small probability of information remaining undiscovered if it exists).

\section{Conclusion}

B\&W's expression (17) and their subsequent analysis is incorrect: It is not true that "..Strategy 1 is optimal for both 'large' and 'small' values of $\pi_{i}$ " (page 189). In this Comment we have shown that Strategy 1 is only optimal for sufficiently small values of $\pi_{i}$.

Condition (23) suggests that Strategy 1 can only be optimal if Regular Investors are, on average, less well-informed than retail investors. Only under this assumption can the Uniform Pricing constraint bind and can the issuer benefit from the ability to price discriminate. Otherwise, when Regular Investors are, on average, at least as well informed as retail investors, in B\&W's set-up we can now rule out the need for price discrimination. In direct contrast, B\&W's equation (17) suggests that whatever the relative informedness of the investor groups, the Uniform Pricing constraint will bind whenever there is a sufficiently high number of regulars, $H$.

\section{Reference}

Benveniste, L.M. and W.J. Wilhelm, (1990), "A comparative analysis of IPO proceeds under alternative regulatory environments", Journal of Financial Economics, 28, 173-20\%. 


\section{Appendix}

\section{A Correcting equation 5}

$$
\begin{gathered}
\frac{\pi}{2}\left(1-\pi_{i}\right)^{H}\left[s^{g}-\left(s^{u}-\varepsilon\right)\right] \beta_{u}+(1-\pi)\left[s^{u}-\left(s^{u}-\varepsilon\right)\right] \beta_{u}+\frac{\pi}{2}\left(1-\pi_{i}\right)^{H}\left[s^{b}-\left(s^{u}-\varepsilon\right)\right]= \\
\frac{\pi}{2}\left(1-\pi_{i}\right)^{H}[\alpha+\varepsilon] \beta_{u}+(1-\pi) \varepsilon \beta_{u}+\frac{\pi}{2}\left(1-\pi_{i}\right)^{H}[\varepsilon-\alpha]=0 \\
\varepsilon\left(\frac{\pi}{2}\left(1-\pi_{i}\right)^{H}+\beta_{u} \frac{\pi}{2}\left(1-\pi_{i}\right)^{H}+\beta_{u}(1-\pi)\right)=\alpha \frac{\pi}{2}\left(1-\pi_{i}\right)^{H}\left(1-\beta_{u}\right) \\
\varepsilon\left(\left(1+\beta_{u}\right)+\beta_{u} \frac{(1-\pi)}{\frac{\pi}{2}\left(1-\pi_{i}\right)^{H}}\right)=\alpha\left(1-\beta_{u}\right) \\
\varepsilon=\frac{\alpha\left(1-\beta_{u}\right)}{\left(1+\beta_{u}\right)+\beta_{u} \frac{2(1-\pi)}{\pi\left(1-\pi_{i}\right)^{H}}}
\end{gathered}
$$

\section{B Correcting equation 6}

Diffentiating our equation (16), B\&W's equation (6) should read

$$
\frac{\partial \varepsilon}{\partial \beta_{u}}=-2 \alpha \pi \frac{\left(\pi+\frac{(1-\pi)}{\left(1-\pi_{i}\right)^{H}}\right)}{\left(\pi\left(1+\beta_{u}\right)+2 \beta_{u} \frac{(1-\pi)}{\left(1-\pi_{i}\right)^{H}}\right)^{2}}
$$

\section{Correcting equations 9 and 10}

$\mathrm{B} \& \mathrm{~W}$ state that "...the expected payoff to an uninformed regular, indicating $\mathrm{u}$ is $(\mathrm{B} \& \mathrm{~W}$ equation 9$)$

$$
E P(u, u)=\begin{gathered}
\frac{\pi}{2} \sum_{h=1}^{H-1} P(g, h)\left[s^{g}-s_{u h}\right] q_{u h} \\
+P(u, 0)\left[s^{u}-s_{u 0}\right] q_{u 0} \\
+\frac{\pi}{2} \sum_{h=1}^{H-1} P(b, h) .0
\end{gathered}
$$

where the $\frac{\pi}{2}$ terms are, presumably, the unconditional probabilities that the true state is good (bad). However, an uninformed regular should update and use the conditional probability of each state, namely

$$
\operatorname{Pr}(\text { state } g \mid \text { uninformed })=\frac{\frac{\pi}{2}\left(1-\pi_{i}\right)}{1-\pi \pi_{i}}
$$

so that

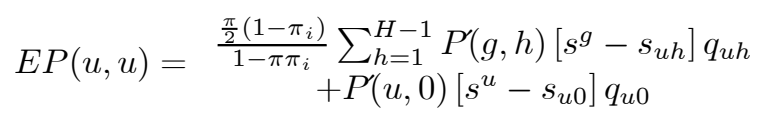

where $P(u, 0)$ is understood to be our corrected expression and the first term can be rewritten as

$$
\frac{1}{1-\pi \pi_{i}} \sum_{h=1}^{H-1} \frac{H-h}{H} P(g, h)\left[s^{g}-s_{u h}\right] q_{u h}
$$


The same error is made in equation 10 .

\section{Correcting equation 17}

our corrected version of equation 15 is our equation (17)

$$
\frac{H P(., 0)}{P(g, 1)} \geqslant \frac{\pi\left(1-\pi_{i}\right)^{H}\left(\beta_{u}+1\right)+2 \beta_{u}(1-\pi)}{\left(1-\beta_{u}\right) \pi\left(1-\pi_{i}\right)^{H}}
$$

By substituting in the explicit expressions

$$
P(., 0)=\left(\pi\left(1-\pi_{i}\right)^{H}+(1-\pi)\right)
$$

and

$$
P(g, 1)=\frac{\pi}{2} H \pi_{i}\left(1-\pi_{i}\right)^{H-1}
$$

we can simplify this to

$$
\begin{gathered}
\frac{H\left(\pi\left(1-\pi_{i}\right)^{H}+(1-\pi)\right)}{\frac{\pi}{2} H \pi_{i}\left(1-\pi_{i}\right)^{H-1}} \geqslant \frac{\pi\left(1-\pi_{i}\right)^{H}\left(\beta_{u}+1\right)+2 \beta_{u}(1-\pi)}{\left(1-\beta_{u}\right) \pi\left(1-\pi_{i}\right)^{H}} \\
\frac{2\left(\pi\left(1-\pi_{i}\right)^{H}+(1-\pi)\right)}{\pi_{i}} \geqslant \frac{\pi\left(1-\pi_{i}\right)^{H}\left(\beta_{u}+1\right)+2 \beta_{u}(1-\pi)}{\left(1-\beta_{u}\right)\left(1-\pi_{i}\right)} \\
2\left(\pi\left(1-\pi_{i}\right)^{H}+(1-\pi)\right)\left(1-\beta_{u}\right)\left(1-\pi_{i}\right)-\pi_{i}\left(\pi\left(1-\pi_{i}\right)^{H}\left(\beta_{u}+1\right)+2 \beta_{u}(1-\pi)\right) \geqslant 0 \\
\text { LHS }=\left(2 \pi\left(1-\pi_{i}\right)^{H}-3 \pi\left(1-\pi_{i}\right)^{H} \pi_{i}-2 \beta_{u} \pi\left(1-\pi_{i}\right)^{H}+\beta_{u} \pi\left(1-\pi_{i}\right)^{H} \pi_{i}\right) \\
\quad+\left(2-2 \pi_{i}-2 \beta_{u}-2 \pi+2 \pi \pi_{i}+2 \beta_{u} \pi\right) \\
\pi\left(1-\pi_{i}\right)^{H}\left(\left(3-\beta_{u}\right)\left(2-\pi_{i}\right)-4\right)+2(1-\pi)\left(1-\pi_{i}-\beta_{u}\right) \geqslant 0
\end{gathered}
$$

which should replace B\&W's equation (17). 